\title{
V.13 ORNL-GM: Development of Ionic Liquid-Additized, GF- 5/6 Compatible Low-Viscosity Oils for Automotive Engine and Rear Axle Lubrication for 4\% Improved Fuel Economy
}

\section{Overall Objectives}

- Further develop ionic liquid (IL)-additized lowviscosity engine oils meeting the GF-5/6 specifications and possessing superior lubricating characteristics

- Expand the IL additive technology to rear axle lubricants

- Seek a combined improvement in the vehicle fuel economy

\section{Fiscal Year (FY) 2016 Objectives}

- Identify the best candidate IL+zinc dialkyldithiophosphate (ZDDP) combination and the optimal treat rate

- Demonstrate $>50 \%$ wear reduction for the IL+ZDDP combination compared with ZDDP alone at the same phosphorus level in bench tests

- Investigate the potential impact of a candidate IL+ZDDP combination on three-way catalysts (TWCs)

\section{FY 2016 Accomplishments}

- We submitted two journal papers and gave two invited talks at technical conferences.

- A phosphorus-containing IL has been identified to have the best synergistic effects with ZDDP when used at 1:1 molecular ratio. When combining the IL with a secondary ZDDP, the optimal treat rate was found to have the P content of 400-800 ppm, which well overlaps with the $600-800 \mathrm{ppm}$ regulated by the GF-5/6 specifications.

- More than $50 \%$ wear reduction was demonstrated by the IL+ZDDP combination compared with the ZDDP alone when added to a polyalphaolefin $4 \mathrm{cSt}$ base oil at the $\mathrm{P}$ level of $800 \mathrm{ppm}$ when tested in boundary lubrication at $150^{\circ} \mathrm{C}$.

- The potential impact of the IL+primary ZDDP combination on TWCs has been investigated. The adverse impact seemed to be minimal.

- A rolling-sliding contact fatigue bench test is being designed to simulate the lifetime heavy-duty cycles

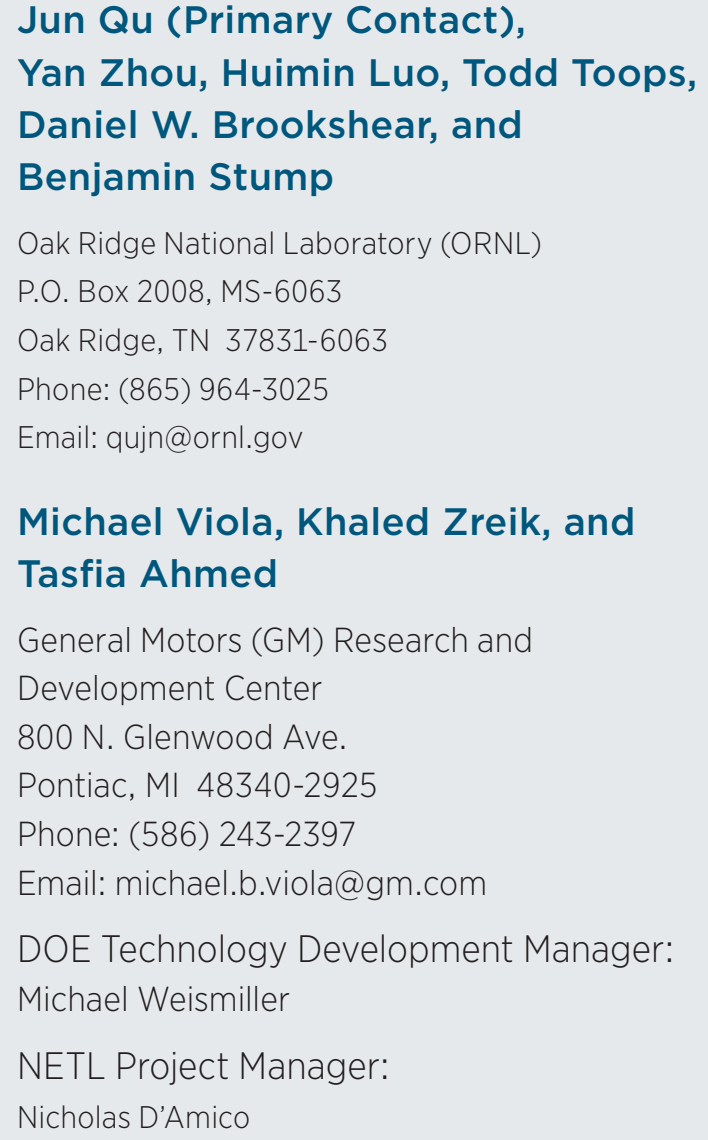

based on contact mechanics and lubrication modeling of the rear axle key contact interface.

\section{Future Directions}

- Formulate a new prototype GF-5/6 SAE 0W-12 engine oil using the top-performing IL+ZDDP combination as the antiwear additive

- Conduct tribological bench tests and engine dynamometer tests to evaluate the improvement on wear protection and fuel economy

- Develop an IL-additized low-viscosity rear axle fluid and demonstrate improved durability and efficiency 


\section{Introduction}

For an automotive vehicle, $10-12 \%$ fuel energy is lost to the parasitic friction in the engine and another $3-5 \%$ must overcome the friction in the rear axle [1,2]. The automotive industry has been applying increasingly lower viscosity oils for both the engine and rear axle to improve fuel economy. The concern in further viscosity reduction is accelerated wear due to thinner lubricant films. Developing more effective anti-wear additives to allow the use of lower viscosity oils has proven to be the most successful and cost effective route. Based on our previous success [3-5] in developing oil-soluble ILs as lubricant additives, the ORNL-GM team is committed in this joint effort to further develop the ILs lubrication technology for both the engine and rear axle to gain a combined improvement in the vehicle fuel economy.

\section{Approach}

Work scope includes: (1) further development of IL-additized low-viscosity engine oils meeting the International Lubricants Standardization and Approval Committee GF-5/6 specifications [6], specifically, investigating the synergy between IL and ZDDP and seeking the optimal treat rate, evaluating the impact of IL+ZDDP on TWCs, formulating a prototype GF5/6 IL-additized multi-grade low-viscosity engine oil, conducting tribological bench tests and analyses, and performing multi-cylinder engine dynamometer tests; (2) development of IL-additized low-viscosity rear axle lubricants, with efforts focused on establishing a contact fatigue bench test to simulate the lifetime duty cycles of rear axle lubrication, tailoring IL chemistry, formulating a prototype IL-additized rear axle fluid, and conducting bench tests and analyses; and (3) executing vehicle tests to demonstrate the improved fuel economy.

\section{Results}

The ORNL-GM team kicked off this joint project on August 4, 2015, and substantial progress has been made in the first year with all milestones met in a timely manner. Based on findings in previous projects, several candidate groups of phosphorus-, sulfur-, or boroncontaining ILs have been synthesized. By comparing the physicochemical and tribological properties, a phosphonium-based IL has been identified as the top candidate because of its high oil-solubility, high thermal stability, and synergistic effects with ZDDP in wear protection and friction reduction.

In order to determine the optimal IL:ZDDP ratio, IL+ZDDP combinations with three molecular ratios, $1: 2,1: 1$, and 2:1, were added to a polyalphaolefin (PAO)
$4 \mathrm{cSt}$ base oil for tribological bench testing. Tests were conducted using an American Iron and Steel Institute 52100 steel ball rubbing against a CL35 cast iron flat at $150^{\circ} \mathrm{C}$ under $100 \mathrm{~N}$ load and $10 \mathrm{~Hz}$ oscillation of $10-\mathrm{mm}$ stroke for 1,000 m sliding. All IL+ZDDP combinations effectively reduced wear by one order of magnitude compared with the neat base oil, and the 1:1 mix of $\mathrm{IL}+\mathrm{ZDDP}$ was the best performer with additional $70 \%$ wear reduction compared with using the ZDDP alone, as shown in Figure 1.

Further investigation on the 1:1 mix of IL+ZDDP in the PAO base oil was then conducted with two types of ZDDPs, primary and secondary, in a wide range of treat rates to understand the trends of the friction and wear behavior. Results are summarized in Figure 2. The coefficient of friction and wear rate (average of two to three repeat tests) are plotted against the $\mathrm{P}$ content in the oil. For IL+secondary ZDDP, the P content of 400-800 ppm seems to have the optimum wear protection (see Figure 2a), which well overlaps with the $600-800 \mathrm{ppm} P$ content regulated by the GF-5/6 specifications. Selected scanning electron microscope images and energy-dispersive X-ray spectroscopy spectra of the worn wears are shown in Figure 3. The morphology and composition of the tribofilm formed in the oil with $400 \mathrm{ppm}$ or $800 \mathrm{ppm}$ of $\mathrm{P}$ seem to be distinct from those produced in other concentrations either higher or lower. Particularly, more $\mathrm{Zn}$ and $\mathrm{P}$ but less $\mathrm{S}$ were detected in the tribofilms with better wear performance. For IL+primary ZDDP, the P content of 800-1,600 ppm seems to perform the best, as shown in Figure 2b. This shift in the optimal concentration may be attributed to the lower reactivity of the primary ZDDP compared with the secondary ZDDP.

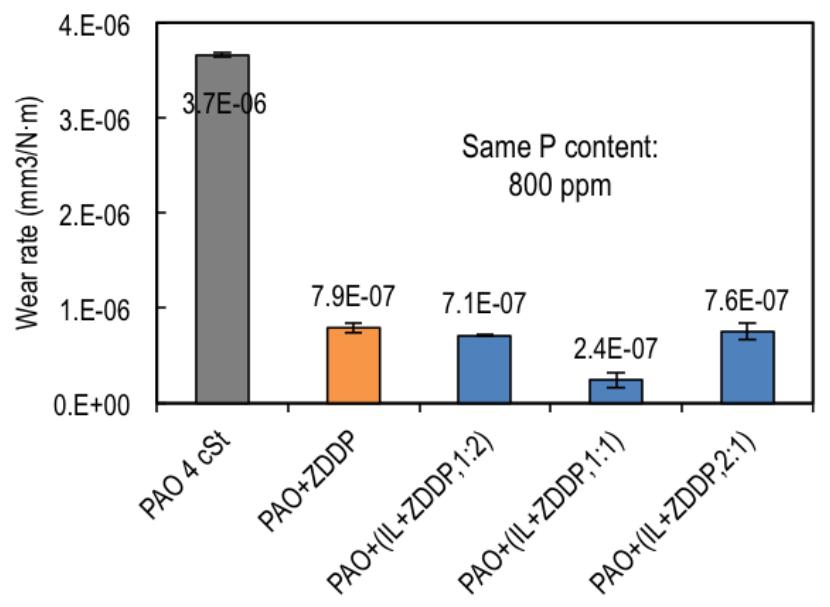

Figure 1. Wear rates using IL+ZDDP combinations of different molecular ratios as oil additives compared with that of the ZDDP alone at the same phosphorus content. 


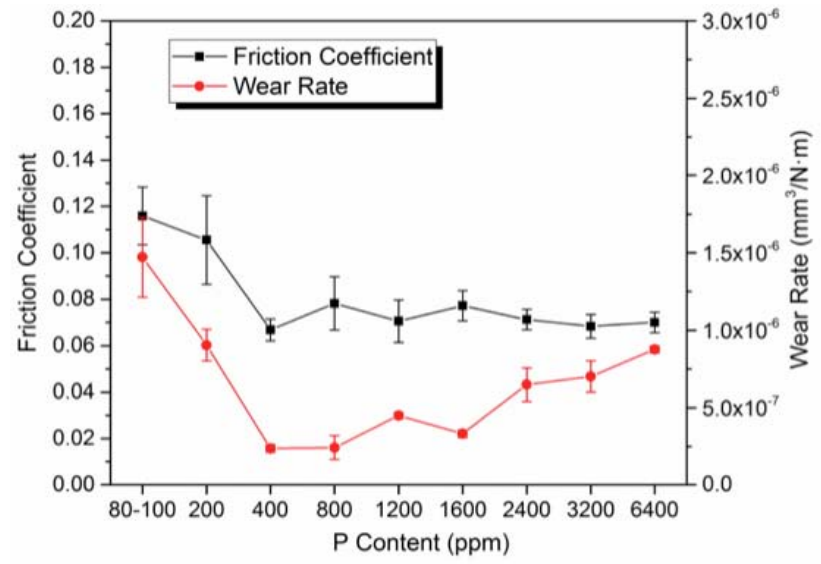

(a) IL+secondary ZDDP [1:1 ratio]

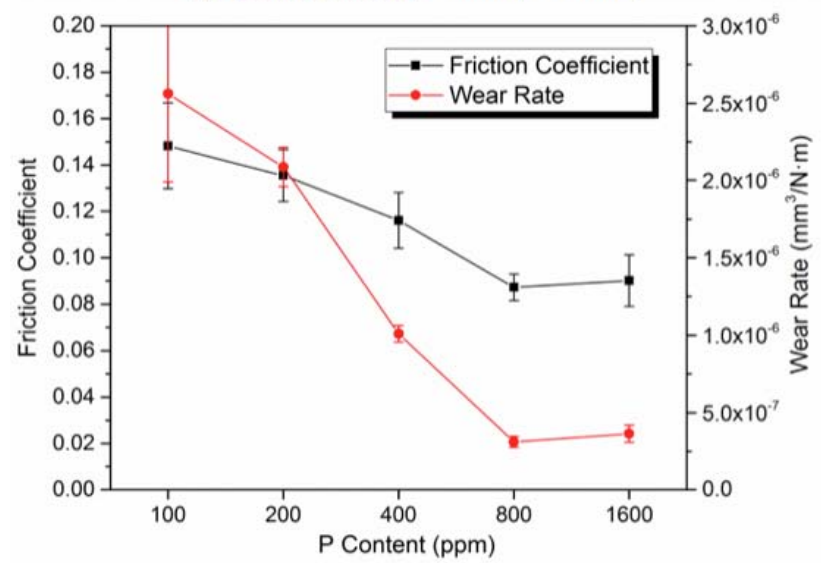

(a) IL+primary ZDDP [1:1 ratio]

Figure 2. Friction and wear results of IL+ZDDP combinations when mixed into a PAO $4 \mathrm{cSt}$ base oil at different concentrations. $400-800$ ppm and $800-1,600$ ppm of $P$ provided the best wear protection for IL+secondary ZDDP and IL+primary ZDDP, respectively.

Stribeck curve multi-scans were conducted using a Mini Traction Machine. The curves were generated in two lubricants containing ZDDP and IL, respectively, at the same amount of $\mathrm{P}(800 \mathrm{ppm})$, under $75 \mathrm{~N}$ at $100^{\circ} \mathrm{C}$ with a $100 \%$ sliding rolling ratio ( $200 \%$ for pure sliding). As compared in Figure 4, the Stribeck curve of the ZDDP-additized oil shifted higher from the first to the fourth scan and then stabilized. The increase in friction was associated with the formation of a ZDDP tribofilm that is known to be a rough and high-friction film. In contrast, the Stribeck curve of the IL-additized oil started $30 \%$ lower compared to that of the ZDDP-additized oil in the first scan and had little friction increase over the following 19 scans. By the end of the 20 scan tests, the Stribeck curve of the IL-additized oil had a substantial friction reduction by as much as $60 \%$ compared to the ZDDP-additized oil. In similar tests, various IL+ZDDP

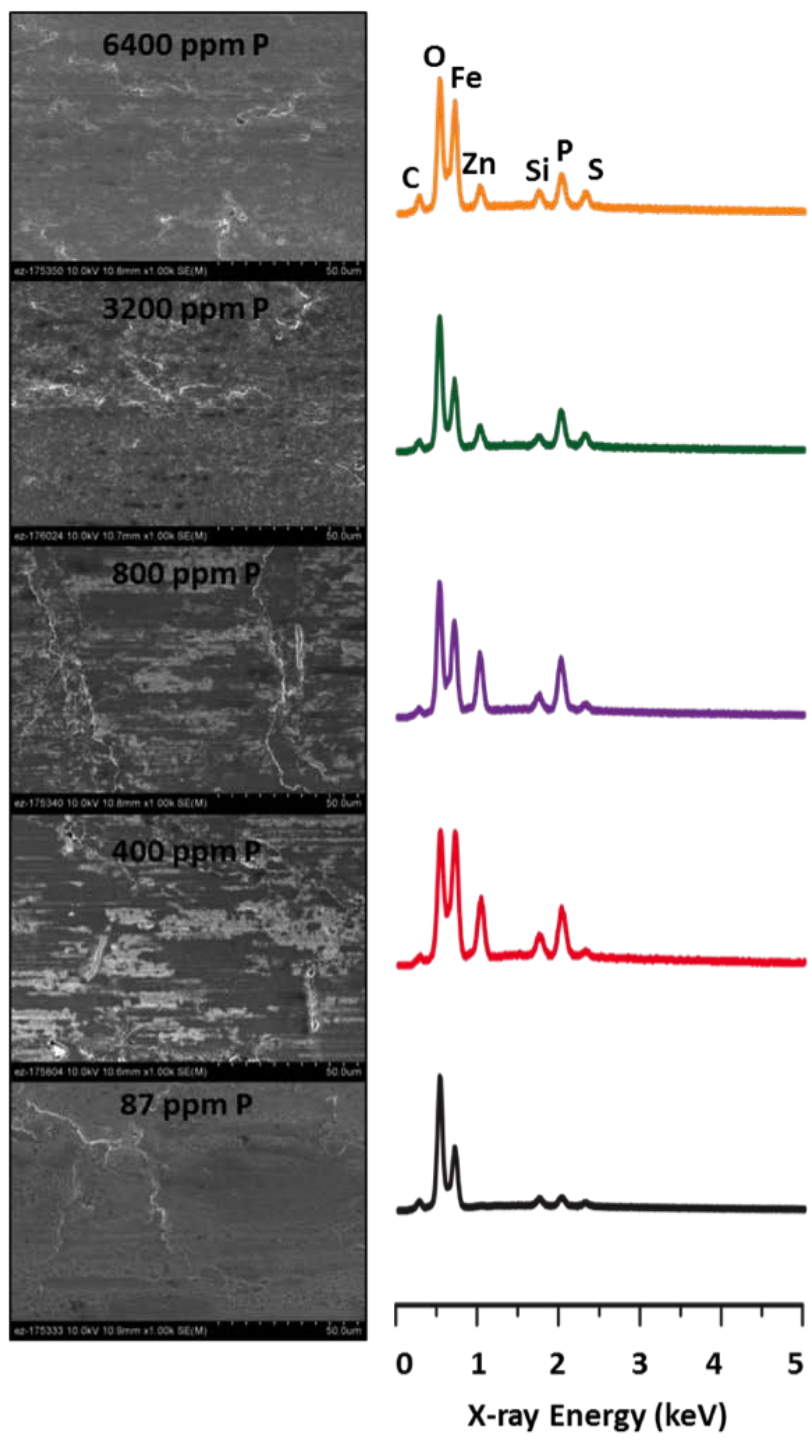

Figure 3. Scanning electron microscopy images and energydispersive X-ray spectroscopy spectra of the worn surface of the cast iron flats lubricated by PAO containing various concentrations of IL+secondary ZDDP (the scale bar represents $20 \mu \mathrm{m}$ ).

combinations also produced lower boundary and mixed friction compared with the ZDDP alone.

The impact of IL+secondary ZDDP on TWCs had been revealed in our previous work [7]. This study investigated the effect of the IL+primary ZDDP combination on TWCs using a similar approach based on accelerated small engine testing. Tests using a fresh 2009 Jeep Liberty TWC were compared among no additive, primary ZDDP, $\mathrm{IL}$, and IL+primary ZDDP. Additive introduction was performed using a stoichiometric gasoline genset with the additive mixed with the fuel. Cores from the front $25 \mathrm{~mm}$ of the TWCs were removed and evaluated in 


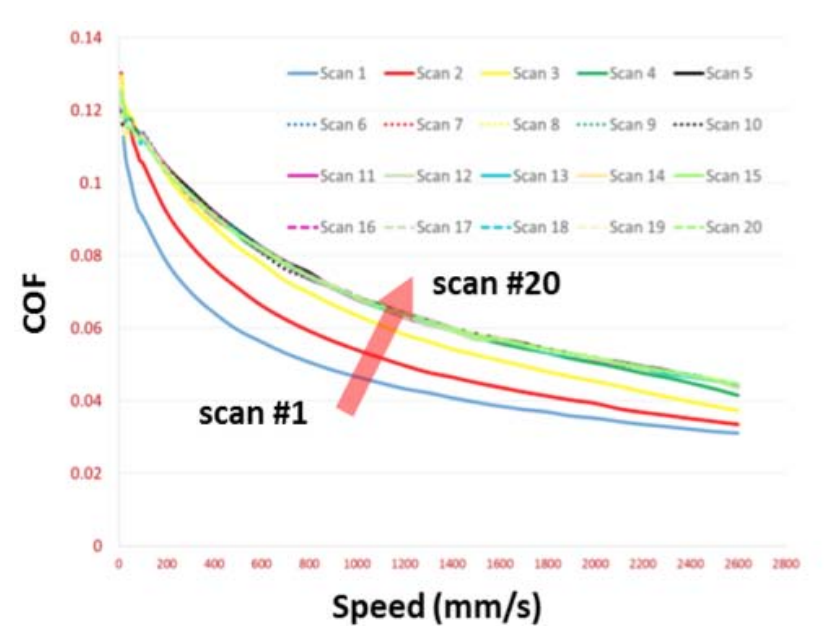

(a) PAO + ZDDP (800 ppm P)

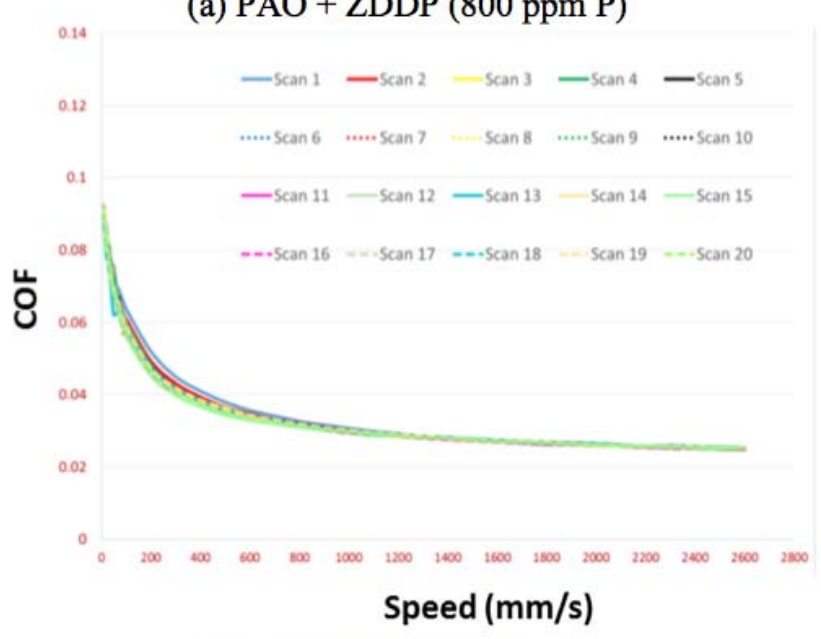

(b) PAO + IL (800 ppm P)

COF - coefficient of friction

Figure 4. Stribeck curves of 20 scans for the PAO 4 cSt base oil containing ZDDP or IL, at the same phosphorous content. The IL generated a 30\% lower friction in the first scan and $30-60 \%$ lower friction in the final scan compared to the ZDDP.

flow reactors. A simulated exhaust mixture containing $\mathrm{CO} / \mathrm{NO}_{\mathrm{x}} / \mathrm{C}_{3} \mathrm{H}_{6}$ (propylene) $/ \mathrm{C}_{3} \mathrm{H}_{8}$ (propane) and a stoichiometric concentration of $\mathrm{O}_{2}$ was introduced to the catalyst cores in the flow reactor and the temperature was ramped from $100^{\circ} \mathrm{C}$ to $600^{\circ} \mathrm{C}$ while measuring the effluent gases. The temperatures of $50 \%$ (T50) and $90 \%$ (T90) conversion are shown in Figure 5. In general, the reactivity was similar for all of the TWCs tested with the three additives. It should be noted that these evaluations are performed after removing the soot and sulfur from the TWCs, and thus are designed to highlight the impact of the additives. Results suggested that the selected IL+secondary ZDDP combination had minimal adverse impact on TWCs.
A pair of rear axle pinion and ring has been ordered and received from a GM supplier, as shown in Figure 6. Both components are made of carburization case-hardened steel with a manganese-phosphate surface coating. Based on a duty cycle load and speed data from GM, the Hertzian contact stress and lubrication regime (lambda ratio, based on Hamrock and Dowson's formula) have been determined for each given operation condition. We are developing a bench test using a micropitting rig to simulate the lifetime heavy-duty cycles experienced by the actual rear axles in a vehicle with matched profiles of contact stresses and lubrication regimes. Initial tests have demonstrated the ability of reproducing the typical micropitting damage (see Figure 6) and distinguishing lubricants in surface protection.

\section{Conclusions}

Significant progress has been made in the first year of the joint project between ORNL and GM. A top-performing candidate IL has been identified and the optimal molecular ratio mixing with ZDDP was determined to be 1:1. The treat rate with maximum friction and wear reductions for IL+secondary ZDDP were found to be in the range of $0.46-0.92 \mathrm{wt} \%$, or $400-800 \mathrm{ppm} \mathrm{P}$, meeting the 800 ppm maximum specified by the GF-5/6. The IL+ZDDP combination has demonstrated $>50 \%$ wear reduction compared with the ZDDP alone when added to a lowviscosity base oil at the $\mathrm{P}$ content of $800 \mathrm{ppm}$ in boundary lubrication. The adverse impact of the IL+primary ZDDP combination on TWCs seemed to be minimal. In addition to engine lubrication, the exploration of potential application of ILs in rear axle lubrication has started with a rolling-sliding contact fatigue bench test to simulate the lifetime heavy-duty cycles of the actual rear axle.

\section{References}

1. S.C. Tung, M.L. McMillan, "Automotive tribology overview of current advances and challenges for the future," Tribology International 37 (2004) 517-536.

2. K. Holmberg, P. Andersson, A. Erdemir, "Global energy consumption due to friction in passenger cars," Tribology International 47 (2012) 221-234.

3. J. Qu, D.G. Bansal, B. Yu, J. Howe, H. Luo, S. Dai, H. Li, P.J. Blau, B.G. Bunting, G. Mordukhovich, D.J. Smolenski, "Anti-wear performance and mechanism of an oil-miscible ionic liquid as a lubricant additive," ACS Applied Materials \& Interfaces 4 (2012) 997-1002.

4. J. Qu, H. Luo, M. Chi, C. Ma, P.J. Blau, S. Dai, M.B. Viola, "Comparison of an oil-miscible ionic liquid and ZDDP as a lubricant anti-wear additive," Tribology International 71 (2014) 88-97. 


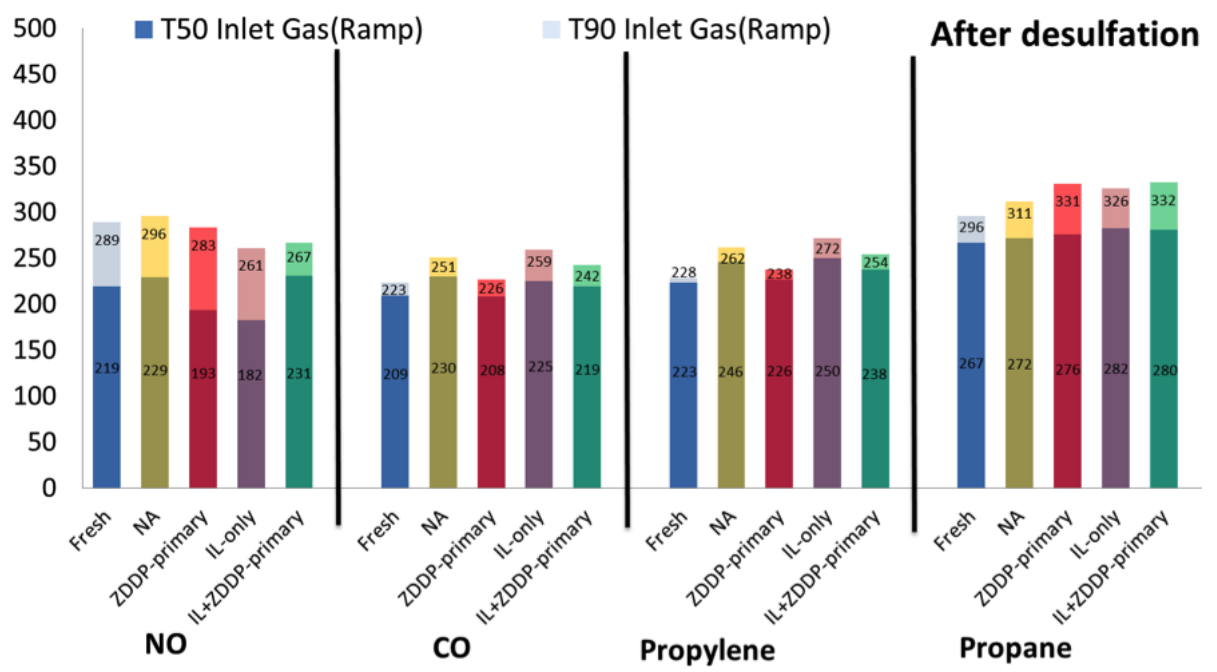

Figure 5. Impact of the primary ZDDP, IL, and IL+primary ZDDP on the TWC (T50 and T90) seemed to be minimal compared to the fresh and no additive (NA) samples.

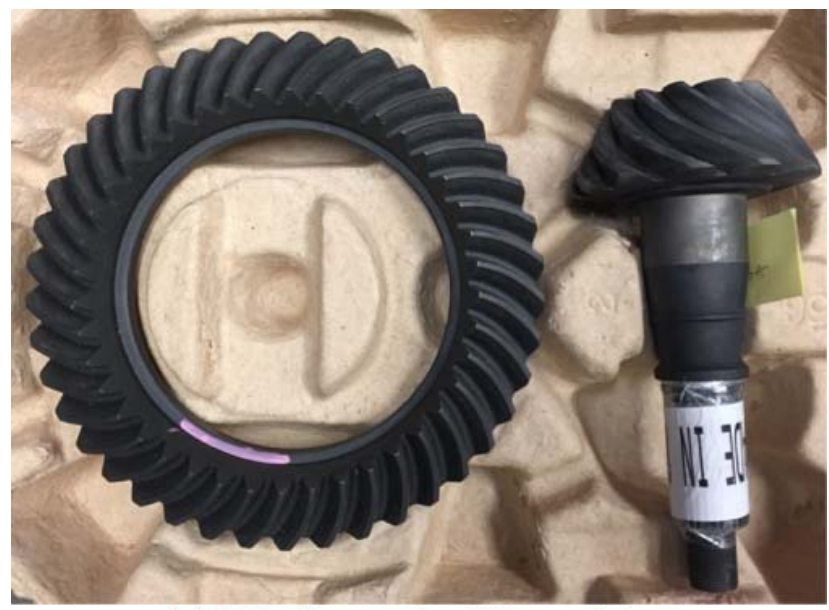

(a) Pair of rear axle pinion and ring

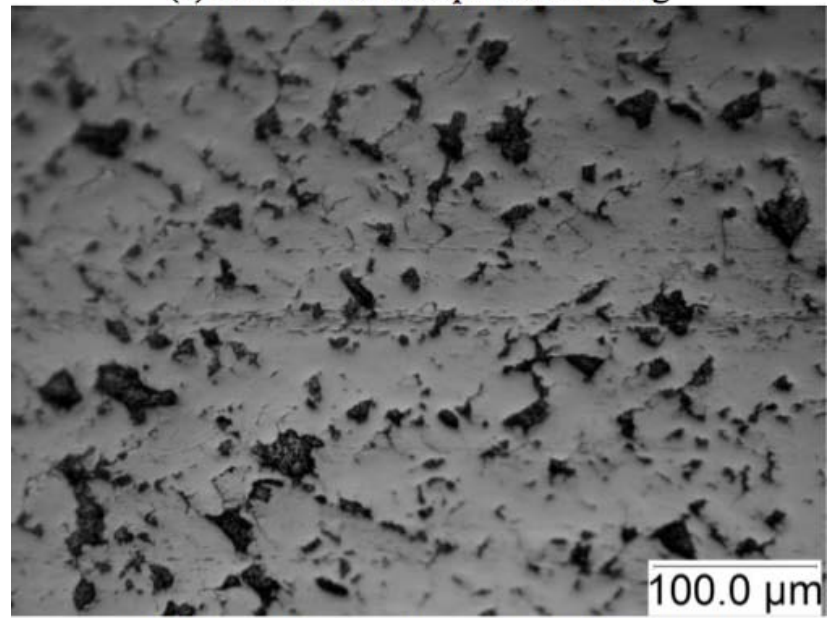

(b) Micro-pitting produced in the bench test

Figure 6. Pair of rear axle pinion and ring and micro-pitting (typical surface damage of axle) produced in the bench test.
5. J. Qu, W.C. Barnhill, H. Luo, H.M. Meyer, D.N. Leonard, A.K. Landauer, B. Kheireddin, H. Gao, B.L. Papke, S. Dai, Synergistic effects between phosphonium-alkylphosphate ionic liquids and ZDDP as lubricant additives, Advanced Materials 27 (2015) 4767-4774.

6. ILSAC Oil Specifications http://www.oilspecifications. org/ilsac.php

7. C. Xie, T. Toops, M. Lance, J. Qu, M. Viola, S. Lewis, D. Leonard, E. Hagaman, "Impact of lubricant additives on the physicochemical properties and activity of three way catalysts," Catalysts 6 (2016) 54.

\section{FY 2016 Publications/Presentations}

1. C. Xie, T. Toops, M. Lance, J. Qu, M. Viola, S. Lewis, D. Leonard, E. Hagaman, "Impact of lubricant additives on the physicochemical properties and activity of three way catalysts," Catalysts 6 (2016) 54.

2. Y. Zhou, J. Qu, "Ionic liquids as lubricant additives - a review," ACS Applied Materials \& Interfaces (in press).

3. J. Qu, "Ionic liquids as novel lubricant additives and their compatibility with other lubricant additives and non-ferrous materials," 2016 Tribology Gordon Research Conference, Lewiston, ME, June 26-July 1, 2016. (Invited)

4. J. Qu, "Oil-miscible ionic liquids as multi-functional additives for low-viscosity engine lubricants," 20th International Colloquium Tribology, Stuttgart, Germany, January 12-14, 2016. (Invited) 